J. Dairy Sci. 97:7905-7915

http://dx.doi.org/10.3168/jds.2014-8125

(C) American Dairy Science Association ${ }^{\circledR}, 2014$. Open access under CC BY-NC-ND license.

\title{
On the value of the phenotypes in the genomic era
}

\author{
O. Gonzalez-Recio, ${ }^{*} \dagger$ M. P. Coffey, $\ddagger$ and J. E. Pryce ${ }^{*}+\S^{1}$ \\ *Biosciences Research Division, Department of Environment and Primary Industries, Agribio, 5 Ring Road, Bundoora, Victoria 3083, Australia \\ †Dairy Futures Cooperative Research Centre, Bundoora, Victoria 3083, Australia \\ ¥Scotland's Rural College (SRUC), Easter Bush Campus, Midlothian, United Kingdom, EH25 9RG \\ $\S$ La Trobe University, Bundoora, Victoria 3083, Australia
}

\section{ABSTRACT}

Genetic improvement programs around the world rely on the collection of accurate phenotypic data. These phenotypes have an inherent value that can be estimated as the contribution of an additional record to genetic gain. Here, the contribution of phenotypes to genetic gain was calculated using traditional progeny testing (PT) and 2 genomic selection (GS) strategies that, for simplicity, included either males or females in the reference population. A procedure to estimate the theoretical economic contribution of a phenotype to a breeding program is described for both GS and PT breeding programs through the increment in genetic gain per unit of increase in estimated breeding value reliability obtained when an additional phenotypic record is added. The main factors affecting the value of a phenotype were the economic value of the trait, the number of phenotypic records already available for the trait, and its heritability. Furthermore, the value of a phenotype was affected by several other factors, including the cost of establishing the breeding program and the cost of phenotyping and genotyping. The cost of achieving a reliability of 0.60 was assessed for different reference populations for GS. Genomic reference populations of more sires with small progeny group sizes (e.g., 20 equivalent daughters) had a lower cost than those reference populations with either large progeny group sizes for fewer genotyped sires, or female reference populations, unless the heritability was large and the cost of phenotyping exceeded a few hundred dollars; then, female reference populations were preferable from an economic perspective.

Key words: phenotype, phenomics, genomic selection

\section{INTRODUCTION}

Data are of paramount importance in dairy breeding programs, and genomic selection and the affordability

Received March 11, 2014.

Accepted September 2, 2014.

${ }^{1}$ Corresponding author: jennie.pryce@depi.vic.gov.au of fully automated and self-contained milk recording systems on farms are potentially threatening to the availability of nationally collated data for genetic evaluations. Most countries have a tradition of collecting milk production records (and other records such as health and fertility). These data are owned by farmers but generally recorded and collected by milk recording organizations (MRO). Additional data, such as conformation and pedigree, are often recorded and managed by breed societies. Genetic evaluations are generally performed by a third party that collates the data. Genetic evaluations have therefore traditionally been an added value by-product of milk recording. However, in recent years, the number of farmers participating in official milk-testing programs has declined in some countries (Figure 1) for several reasons, including financial hardship, lack of time for or interest in participating in an MRO, or the growth in automated milk recording systems available on-farm. Consequently, for some farms, there is no longer the requirement to involve an $\mathrm{MRO}$ in record-keeping; therefore, unless an alternative arrangement is made for data transfer, these data would not be available for genetic evaluation units that rely on the MRO for their data supply.

Furthermore, the affordability of large-scale SNP genotyping and the construction of worldwide consortia for the exchange of genotypes and genomic selection (GS) have helped to create reference populations large enough to produce reliabilities close to 0.70 for traits with high heritabilities (Pryce and Daetwyler, 2012; Ducrocq and Wiggans, 2014). This has favored the use of young non-progeny-tested bulls, thereby reducing the necessity of progeny testing and milk recording for genetic improvement. The market share of young genomic bulls (without daughters) has increased to more than half of dairy inseminations in many countries (Ducrocq and Wiggans, 2014; Hutchison et al., 2014). The problem that has manifested is that this practice threatens the data availability for future reference populations because the possibility exists that insufficient data will be collected in the absence of structured progeny-testing schemes. The success of GS depends on a large reference population of animals that have both 

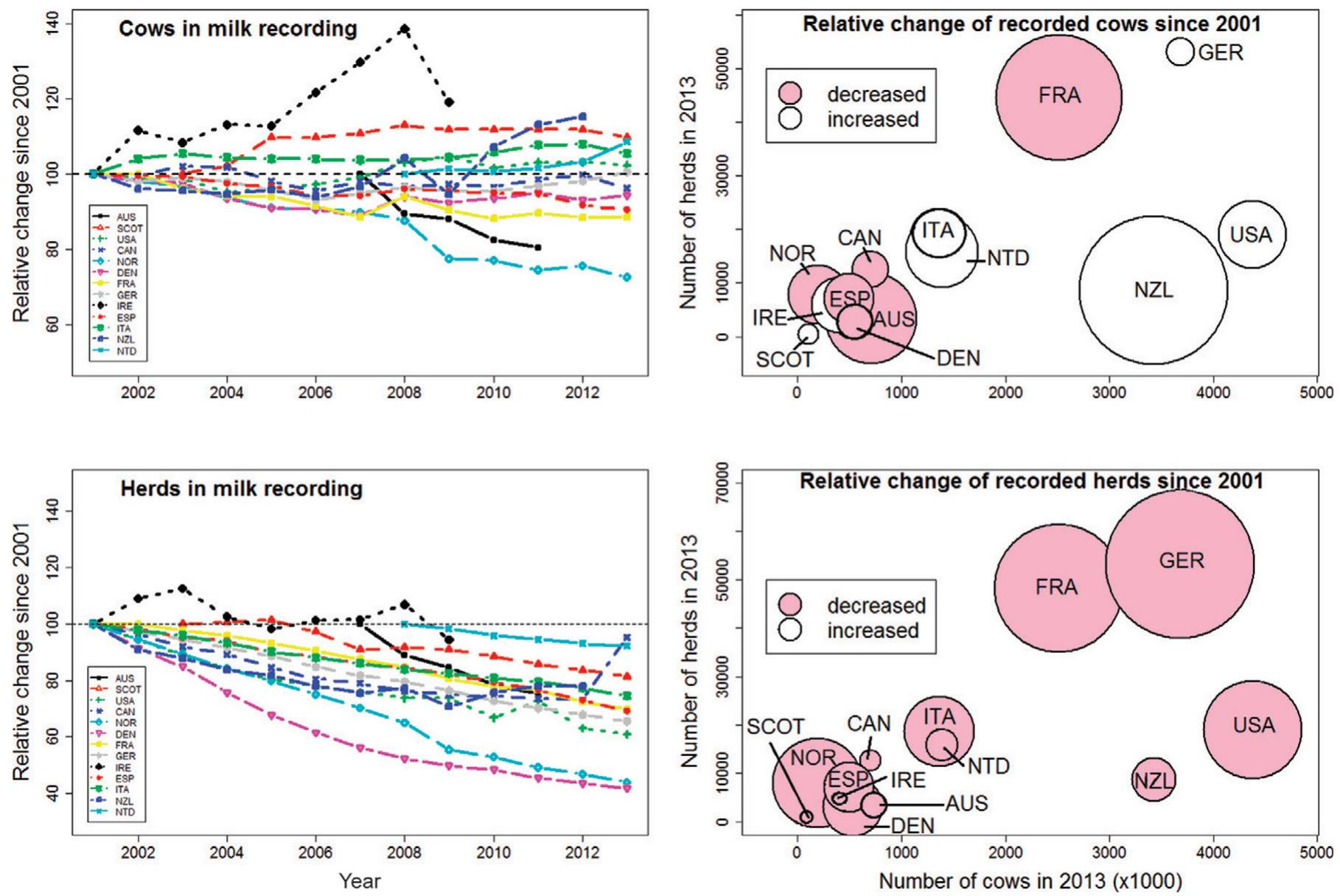

Figure 1. Annual relative change (\%) in the number of cows (upper left) and herds (lower left) involved in the milk recording organizations of different countries since 2001, and the relative change in the number of cows (upper right) and herds (lower right) from 2001 to 2013 (or closest available year) per country regarding the number of cows and herds involved in milk recording organizations in 2013 (larger circles represent larger relative change). AUS = Australia; CAN = Canada; DEN = Denmark; SCOT $=$ Scotland; ESP $=$ Spain; FRA $=$ France; GER $=$ Germany; IRE = Ireland; ITA = Italy; NTD = the Netherlands; NOR = Norway; NZL = New Zealand; USA = United States of America. Source: ICAR (2013). Color version available in the online PDF.

phenotypes and genotypes that are used to predict genomically enhanced breeding values of new individuals. Phenotypes may be an animal's own performance (e.g., commercial cows, or in exceptional cases, experimental cows for rare phenotypes) or progeny performance (e.g., sires). Until recently, the reference populations of most countries that have implemented GS were limited to genotyped bulls, each with a large number of phenotypes from progeny obtained from traditional progeny-testing programs (PT). There is growing interest in adding data from females to the reference population (Pryce and Daetwyler, 2012; Ducrocq and Wiggans, 2014). This trend is likely to continue because as official genomic evaluations become available, the number of bulls being progeny-tested is declining. Regardless of whether males or females are included in the reference population, the size of the reference population is currently limited by the number of animals with genotypes, because the number of phenotyped animals undergoing GS is large for most traits associated with production (e.g., milk yield in dairy cattle, and growth and weight traits in poultry and swine). This may change over time, as genotyping becomes cheaper and the need to participate in milk recording decreases. However, for some novel traits such as feed efficiency, methane emissions, or level of hormones or metabolites, phenotypes are scarce because the traits are expensive or difficult to measure and are therefore rarely available for commercial dairy herds. For example, the cost of phenotyping for feed intake is estimated to be around AU $\$ 1,000 /$ cow per year for a 28-d period (Bill Wales, Department of Environment and Primary Industries, Victoria, Australia; personal communication) or $£ 700 /$ cow per year for a 70-d period (Eileen Wall, SRUC, Edinburgh, UK; personal communication). Hence, these phenotypes are available for only some animals in the 
Table 1. Economic values $(e v)$, heritability $\left(h^{2}\right)$, and additive genetic standard deviations $\left(\sigma_{a}\right)$ of traits used in the analyses

\begin{tabular}{lccc}
\hline & $e v$ & & \\
Trait & (AU\$/unit/yr) & $h^{2}$ & (trait units) \\
\hline Calving interval (d) & -3.02 & 0.05 & 7.97 \\
Residual feed intake (kg of feed) & -102.61 & 0.27 & 0.20 \\
Milk yield (L) & -0.05 & 0.30 & 445.42 \\
Protein yield (kg) & 5.99 & 0.30 & 12.47 \\
Fat yield (kg) & 1.49 & 0.30 & 16.32 \\
\hline
\end{tabular}

population. The benefit of including these traits in a breeding program must justify the investment.

The cost and ultimately the benefit of phenotyping have usually been ignored or not estimated in animal breeding for several reasons: the added value of a phenotype is not straightforward and, until now and for the foreseeable future, phenotypes have been made freely available through milk recording. Phenotypes are not only used for genetic evaluations and breeding, but also for management and financial purposes that accumulate value over time. One possible scenario is that those in control of breeding schemes may have to purchase phenotypes in the future; therefore, a method is required to value each additional phenotype for payment purposes. However, the value of phenotypes is not constant over time for breeding purposes, because their contribution to the accuracy of genetic evaluations depends on the amount of data already available.

Therefore, the following questions arise: What is the contribution of a new phenotype given that several phenotypes are already available? For the same investment in phenotyping, is the best return gained from progeny-testing or genomic selection? If genomic selection is preferred, in what circumstances is it preferable to have either bull or cow reference populations?

In this study, we describe a procedure to estimate the economic contribution of a phenotype to a breeding program for both PT and GS breeding programs, using examples of a single-trait breeding goal. Guidelines for the most cost-effective strategy to establish a genomic reference population considering the phenotyping costs are described.

\section{MATERIALS AND METHODS}

First, we propose a method to estimate the contribution of a single phenotype to the genetic gain made in a population using a traditional breeding program. The counterpart using GS is then calculated for reference populations of (1) genotyped males with progeny groups of daughters, and (2) genotyped females. The cost of establishing a reference population to obtain a reliability of 0.60 is evaluated as a function of the phenotyping cost. Parameters used in the calculations are shown in Table 1.

\section{Economic Contribution of Additional Phenotypes in a Breeding Program}

The sire reliability ( $R e l$ ) given several progeny records available was approximated, as described by Cameron (1997):

$$
R e l=0.5 \times \frac{\frac{1}{2} n h^{2}}{1+(n-1) \frac{1}{4} h^{2}},
$$

where $h^{2}$ is the heritability and $n$ is the progeny size in terms of effective daughter (ED) contribution (Van Raden and Wiggans, 1991; Fikse and Banos, 2001).

Therefore, the contribution of an additional daughter with a record can be calculated by the derivative of the reliability $\left(R e l^{\prime}\right)$ with respect to $n,\left(\frac{\mathrm{d} R e l}{\mathrm{~d} n}\right)$, as follows:

$$
R e l^{\prime}=\frac{\mathrm{d} R e l}{\mathrm{~d} n}=\frac{h^{2}\left(4-h^{2}\right)}{\left[4+(n-1) h^{2}\right]^{2}} .
$$

This can be viewed as the increase in reliability in sire EBV, for a given heritability, from an additional daughter phenotype when there are $n$ daughters with phenotypes already available. Assuming that the increase in reliability is achieved in all sires, it is easy to obtain the corresponding economic contribution to genetic gain achieved in the populations given a certain economic value (Groen, 1990) of the trait (change or benefit per unit of trait). The classic equation to calculate genetic gain $(\boldsymbol{\Delta} \boldsymbol{G})$ in the sire (sire-sire and siredam) paths comes from the following equation (Lush, 1945):

$$
\Delta G=\frac{i \cdot \rho \cdot \sigma_{a}}{L}
$$

where $i$ is the selection intensity, $\sigma_{a}$ is the additive genetic standard deviation, $L$ is the generation interval, 
and $\rho$ is the square root of the reliability. Then, the first derivative of the genetic gain equation with respect to $n,\left(\frac{\mathrm{d} \Delta G}{\mathrm{~d} n}\right)$, provides the contribution to the genetic gain per record added into the genetic evaluations as

$$
\frac{\mathrm{d} \Delta G}{\mathrm{~d} n}=\frac{\mathrm{d} \Delta G}{\mathrm{~d} \rho} \frac{\mathrm{d} \rho}{\mathrm{d} n}=\frac{i \cdot \sigma_{a}}{L} \frac{\mathrm{d} \rho}{\mathrm{d} n}=\frac{i \cdot \sigma_{a}}{L} \frac{h^{2}\left(4-h^{2}\right)}{\rho\left[4+(n-1) h^{2}\right]^{2}} .
$$

Hence, the theoretical economic contribution (in AU\$) of the phenotype from an additional daughter is the increment in genetic gain in the population (in AU\$) obtained at increasing the reliability due to recording an additional phenotype in the population:

$$
e v \cdot \frac{\mathrm{d} \Delta G}{\mathrm{~d} n}
$$

where the economic value $(\boldsymbol{e v})$ of the trait was defined as the marginal variation of the efficiency (in AU\$) of a production system, at the moment of expression of unit of genetic superiority for the trait (Groen, 1990).

Selection intensity and generation interval were assumed to be 2 and 3.5, respectively, in the sire path.

\section{Genomic Prediction}

Daetwyler et al. (2008) proposed that the reliability of genomic prediction in selection candidates could be calculated as follows:

$$
R e l=\frac{N h^{2}}{N h^{2}+q},
$$

where $N$ was the number of individuals genotyped and phenotyped in the reference population, $h^{2}$ is the heritability of the trait or the reliability of breeding values in a sire population, which is $\frac{n}{\frac{4-h^{2}}{h^{2}}+n}$, according to Cameron (1997), where $n$ is the progeny size, $q$ is the number of independent chromosome segments, and $q=$ $2 N_{e} L_{g}$, where $N_{e}$ is the effective population size (assumed to be 100) and $L_{g}$ is the length of the genome in Morgans (assumed to be 30). Daetwyler et al. (2008) have a final correction of the prediction of the reliability which is to add $\left(\operatorname{Rel}+\operatorname{Rel}^{4} q\right) / 2 N$.

The reliability in selection candidates was calculated for female and male reference populations separately. We assumed that reference populations varied in size between 1,000 and 100,000, and only one phenotype was available per female. For the male reference populations, the number of daughters per sire varied between 1 and 50, and the male genomic reference populations varied in size between 1,000 and 50,000.

Then, the first derivative of [2] with respect to $N$ or $n$ provides the contribution to the increase of genomic reliability per unit of change in the number of genotyped animals or progeny group size, respectively. The contribution to genetic gain per unit increase in genomic reliability was calculated as in [1] with the respective $\frac{\mathrm{d} \rho}{\mathrm{d} n}$. In the genomic selection scenarios, $L$ was assumed to be 3 , as this is typical of literature values from breeding scheme designs under GS (see the review of Pryce and Daetwyler, 2012).

The economic gain ( $\mathrm{Val}$ ) of improving an index by 1 genetic standard deviation was calculated as $\mathrm{Val}=\Delta G$ $\times C \times e v$, where $C$ is the number of cows in the population $(500,000)$ and $e v$ is the economic value of the trait as defined above. The value of each phenotype can then be shared over individuals in the reference population to calculate the economic contribution of an additional phenotype to the population: $\operatorname{Val} \times \Delta G / N$.

The comparison between PT and GS was examined assuming that 450 young sires were tested annually, a number chosen because it is the number of bulls that are currently genomically tested in Australia (Ducrocq and Wiggans, 2014).

\section{Relative Cost of Female Versus Sire Reference Populations}

A straightforward way to approximate the cost of a GS program is as a function of phenotypes and genotypes needed in the reference population. The cost of a female-only reference population is

$$
C_{\text {Female }}=N_{F} \cdot g+N_{F} \cdot p
$$

and the cost of a sire reference population is

$$
C_{\text {Sire }}=N_{s} \cdot g+d t r s \cdot N_{s} \cdot p,
$$

where $N_{s}$ and $N_{F}$ are the number of sires or cows needed to achieve accuracy of 0.60 in selection candidates, respectively, $g$ is the cost of genotyping (assumed to be $\mathrm{AU} \$ 60), p$ is the cost of phenotyping ranging between $\mathrm{AU} \$ 0.01$ and $\mathrm{AU} \$ 2,000$ per record, and dtrs is the progeny size in the sire reference population (set to either 20 or 100). We compared the cost of the 3 example reference populations: $C_{\text {Female }}, C_{\text {Sire }}$ for progeny size of 20 daughters, and $C_{\text {Sire }}$ for progeny size of 100 daughters. 


\section{RESULTS}

\section{Economic Contribution of Phenotypes}

In accordance with the results of Daetwyler et al. (2008), higher reliabilities are theoretically expected with larger progeny group sizes for PT or larger reference male or female populations for GS (Figure 2). Progeny group sizes $>150$ are needed to obtain reliabilities of more than $90 \%$ for high heritability traits $\left(h^{2}>0.30\right.$; Figure 2a). This means that 67,500 phenotypes from crops of daughters would be required to achieve a reliability of $90 \%$ through a progeny-testing of 450 young sires. In a genomic reference population that includes only females, $>100,000$ genotyped cows are necessary to obtain equivalent reliabilities in candidate animals (Figure 2b). In fact, this number of phenotypes would be equivalent to progeny testing 665 young sires; however, EBV for any number of genomically tested young bulls is possible with GS once the regression equations are estimated. The same accuracy could also be achieved using a reference population of 20,000 males, with progeny groups of $8 \mathrm{ED}$ each, (Figure 2c), which is a total of 160,000 phenotypes. But this reference population would have higher phenotyping costs than those from traditional PT or female reference populations. The reliability increases by only $3 \%$ between 20 and 50 ED for 20,000 sires in the reference population and $h^{2}=$ 0.50 . For low heritability traits such as calving interval $\left(h^{2}=0.05\right)$, progeny groups of 500 daughter equivalents produce reliabilities of around $85 \%$ in PT breeding programs (i.e., 225,000 phenotypes per 450 progeny-tested bulls), whereas female reference populations comprising the same number of cows would lead to reliability in candidate individuals that is roughly one-third of $85 \%$ for these low heritability traits. For GS with sire reference populations around 20,000 sires, progeny groups of $20 \mathrm{ED}$ are required to achieve reliabilities of $85 \%$ (i.e., a total of 400,000 phenotypes).

It seems that PT needs fewer records than GS to obtain a given reliability; however, this advantage disappears as soon as an additional generation is added, because another PT is needed, whereas only a minor update of the reference population is necessary to continue genomic prediction. Clearly, the main advantage of a genomic reference population is that yearly updates with phenotypes and genotypes can be undertaken at a much lower cost, because a reduced number of individuals are required. Furthermore, any number of young sires can be genomically tested annually. On the other hand, progeny testing needs the same amount of progeny to be phenotyped each year for a constant number of progeny-tested bulls, and every young bull needs to be progeny tested.
To achieve breeding value reliabilities of $65 \%$ (the minimum level that is likely to be acceptable to the dairy industry) for production traits $\left(h^{2}=0.30\right)$ and ignoring contributions from pedigree, it is possible to use large reference populations of sires with reduced progeny group sizes (e.g., 20,000 sires with 2 ED each or 5,000 sires with 6 ED each; Figure 2c). For low heritability traits, reliabilities of $65 \%$ or greater are unlikely to be achieved using GS with solely either female reference populations or bull reference populations constructed with fewer than 2,000 sires.

Female-only reference populations need to be large to achieve the accuracies that are achieved with PT or genomic reference populations comprising males, although in some cases fewer phenotypes are necessary compared with the sire reference population to achieve the same reliability. For instance, for a trait with an $h^{2}$ of 0.30 , a reliability of $87 \%$ is achieved through a progeny test of $80 \mathrm{ED}(36,000$ phenotypes to progeny test 450 young bulls) when only the contribution of daughters is considered (i.e., ignoring the contribution from other ancestors) or using a male genomic reference population of 20,000 individuals each with $19 \mathrm{ED}$ (i.e., 380,000 phenotypes), whereas around 130,000 genotyped females, with 1 record each, are needed to realize the same reliability. For a trait with an $h^{2}$ of 0.05, the progeny-test reliability for $80 \mathrm{ED}(36,000$ phenotypes to progeny test 450 young bulls) would be 0.50 . The same reliability is achievable with a female genomic reference population of 120,000 cows or a male reference population of 20,000 sires with $35 \mathrm{ED}$ each (i.e., 700,000 records), or a male reference population of 7,000 sires with $80 \mathrm{ED}$ each (i.e., 560,000 records).

The theoretical contribution to reliability of an additional phenotype given that $n$ daughters, genotyped cows, or sires have already been phenotyped or progeny proven is shown in Figure 3. As expected, the relative importance of an additional phenotype decreases with the number of daughters of progeny-test sires, sires in the genomic reference population, or females in the reference population. The higher the heritability, the faster the decay; therefore, additional phenotypes contribute less to genetic gain in the population. For instance, in a traditional progeny-testing scheme that includes traits with $h^{2}$ of 0.50 and 0.30 , once the progeny group size reaches 120 and 200 ED, respectively, an additional daughter per sire does not have much effect on reliability; therefore, their contribution to the genetic gain in the population is zero (Figure 3a). Similarly, in a GS program of cows, the contribution of each additional phenotyped cow decays until 100,000 individuals, regardless of the heritability. However, the larger the heritability, the larger the decay in contribution as records increase. Smaller changes were observed 
Traditional (a)

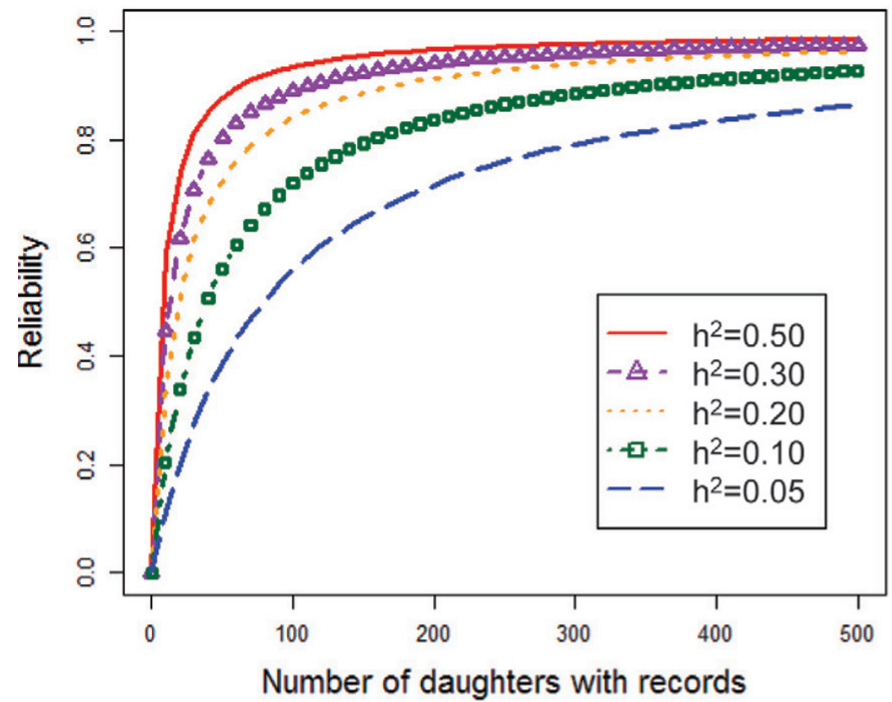

Genomic selection - males (c.1)

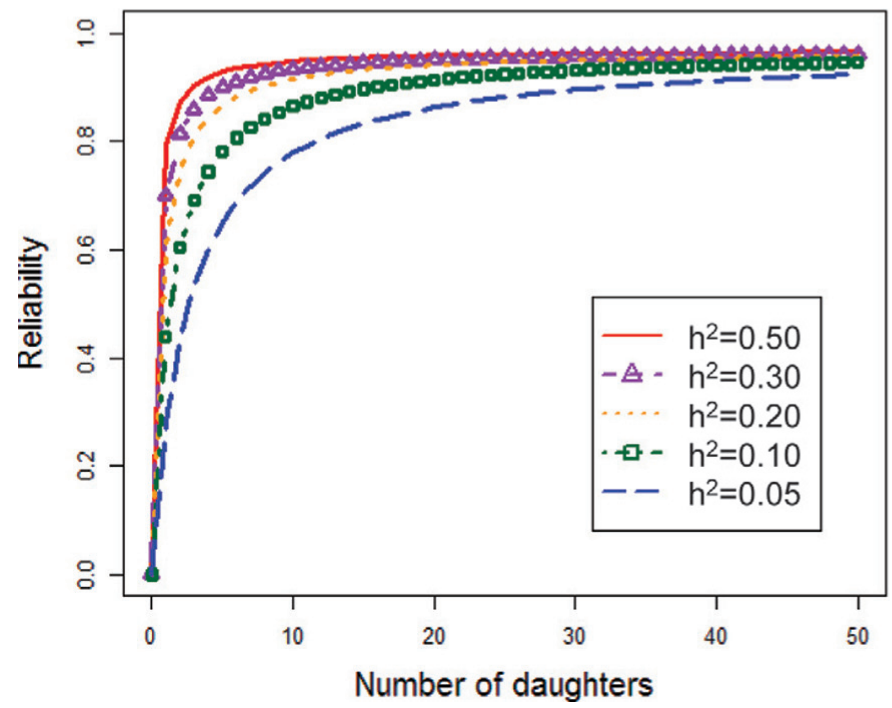

Genomic Selection - females (b)

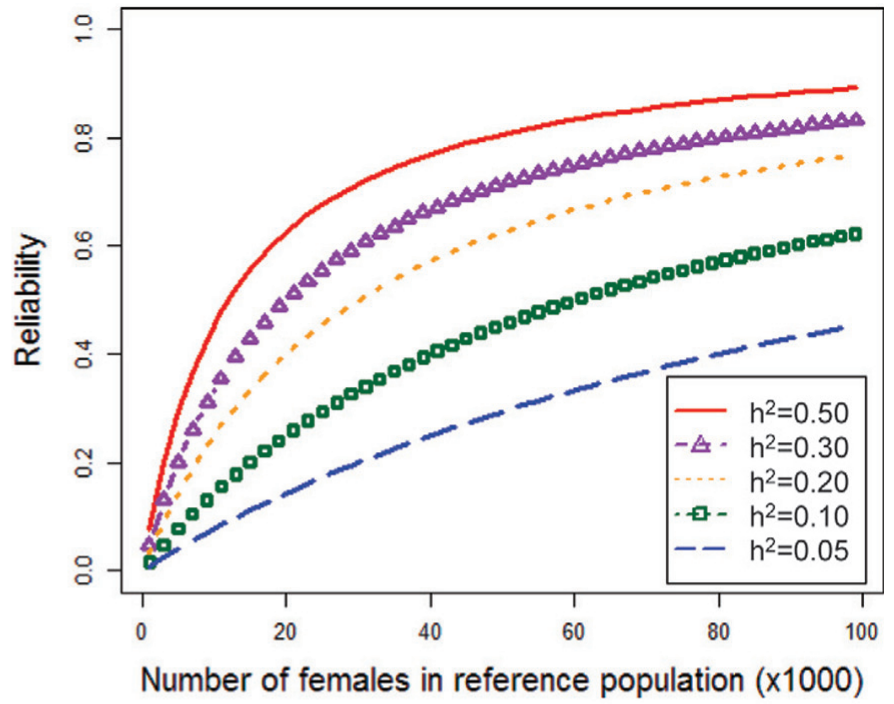

Genomic selection - sires $\left(h^{2}=0.30\right)(c .2)$

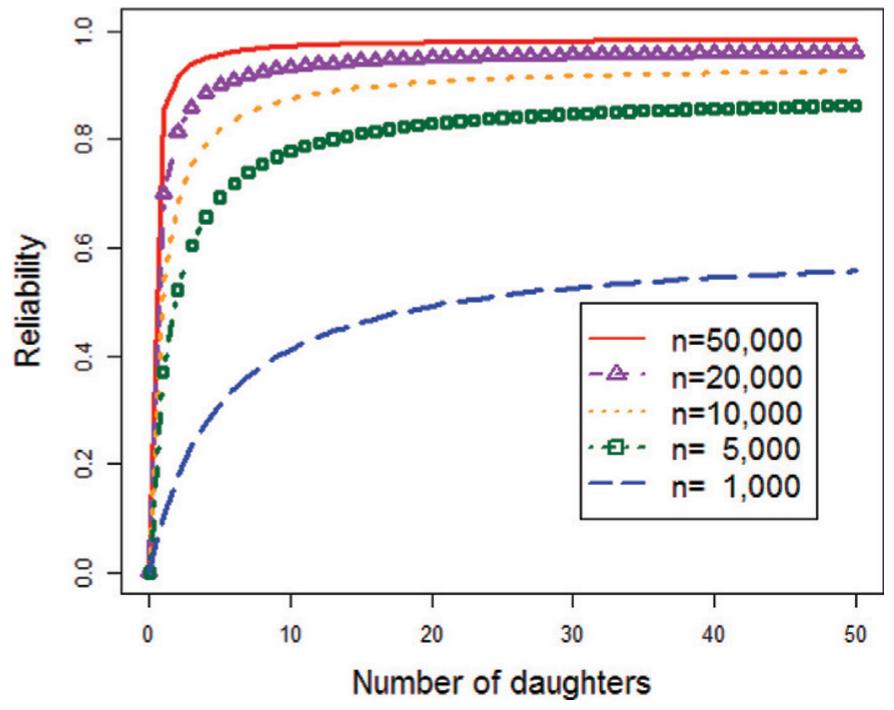

Figure 2. Expected reliability of genetic prediction in candidate animals according the number of progeny with records and the heritability $\left(h^{2}\right)$ of the trait in (a) a traditional breeding program, (b) a genomic selection with a female reference population, (c.1) genomic selection with a male reference population of 20,000 and different progeny group sizes, or (c.2) genomic selection in a sire reference population for a trait with heritability of 0.30 . Color version available in the online PDF.

for lower heritability traits, and similar values for each additional phenotype were observed (i.e., more constant decay). In sire reference populations, the trend was similar to that in PT programs. For traits with lower heritability, such as fertility (e.g., 0.05), progeny group sizes up to $500 \mathrm{ED}$ may be needed in a progenytest scheme until each additional phenotype no longer affects the contribution to reliability.

Figure 4 shows the theoretical economic contribution to the Australian dairy industry of an additional phe- notype for the traits included in the study. It can be seen that in the early stages of a PT breeding program, when limited phenotypic data are available, it is better to obtain phenotypes for traits with larger economic weights in a selection index (e.g., calving interval or protein yield), regardless of whether the selection is performed via PT or GS. The economic contribution of phenotypes is lower for low heritability traits (e.g., fertility) than for production traits. However, the decay in economic contribution of low heritability traits is 

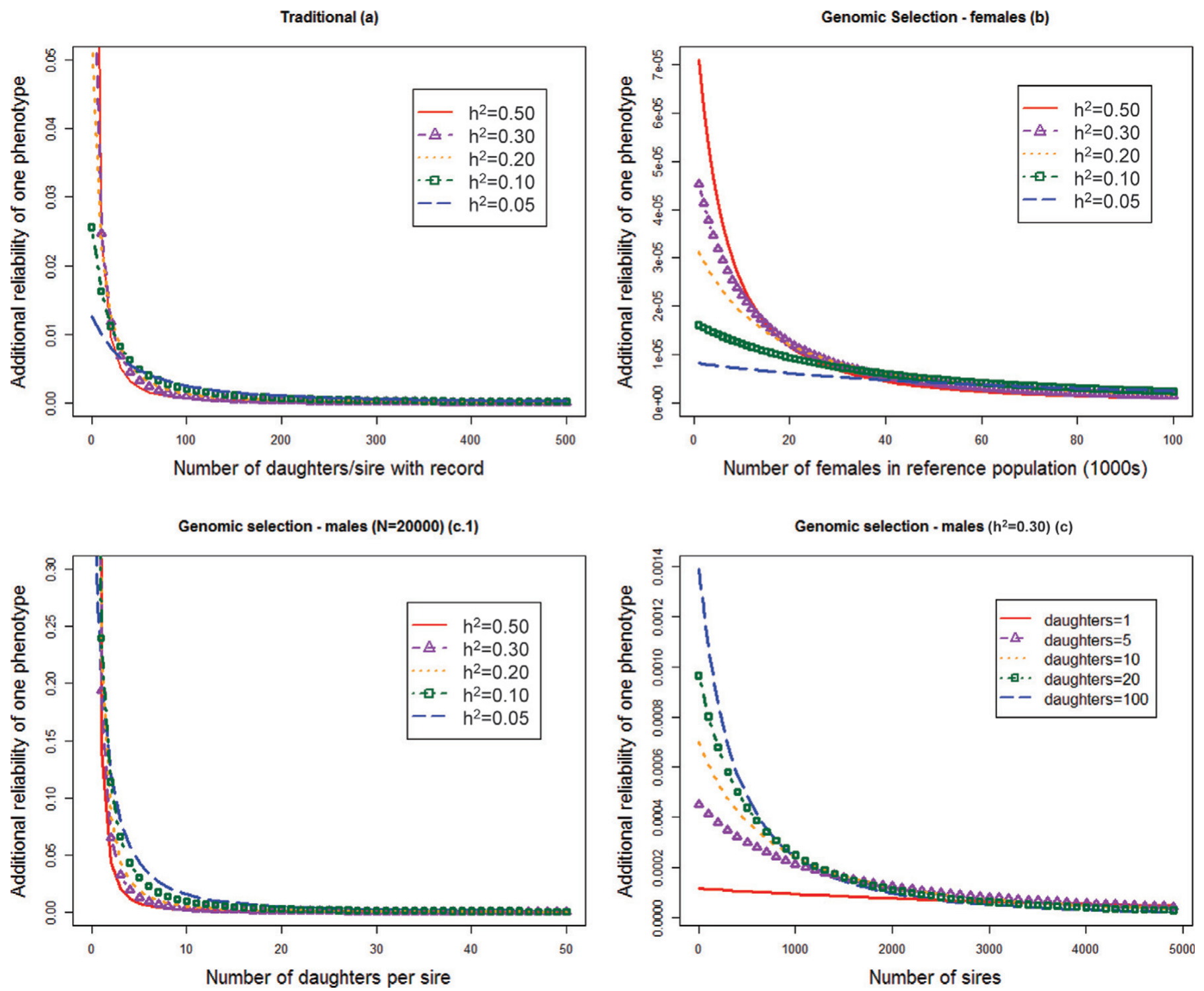

Figure 3. Expected contribution to the increase in reliability of an additional phenotype given that cows have already been phenotyped in (a) a traditional breeding program, (b) genomic selection with a female reference population, (c.1) genomic selection with a male reference population of 20,000 and different progeny sizes, or (c.2) genomic selection in a sire reference population for a trait with heritability of 0.30 . Color version available in the online PDF.

slower, which means that more phenotypes are needed until the contribution of new phenotypes is negligible. In a traditional PT program, when more than $50 \mathrm{ED}$ are phenotyped for all traits, the economic contribution of an additional phenotype for calving interval is larger than an extra phenotype for milk yield. Also, when $100 \mathrm{ED}$ are phenotyped for protein yield, but no calving interval phenotype is available, the contribution of one daughter with a fertility phenotype per sire is $81 \%$ larger than that of an additional daughter per sire with PY phenotype. The same trend is observed for the GS population; however, the decay of low heritability traits is faster in female reference populations, probably because the reliability that can be obtained with a cow reference population is limited, as shown in Figure 2. As expected, the relative importance of liquid milk is less than that of protein yield because of the larger economic weight of the latter in Australia, which is consistent with most countries. Traits with high heritability estimates but small economic values rapidly decay in their economic contribution to population genetic gain as more progeny or reference animals with phenotypes are available. Figure $4 \mathrm{c}$ shows that when a large sire reference population is already established (e.g., 20,000 

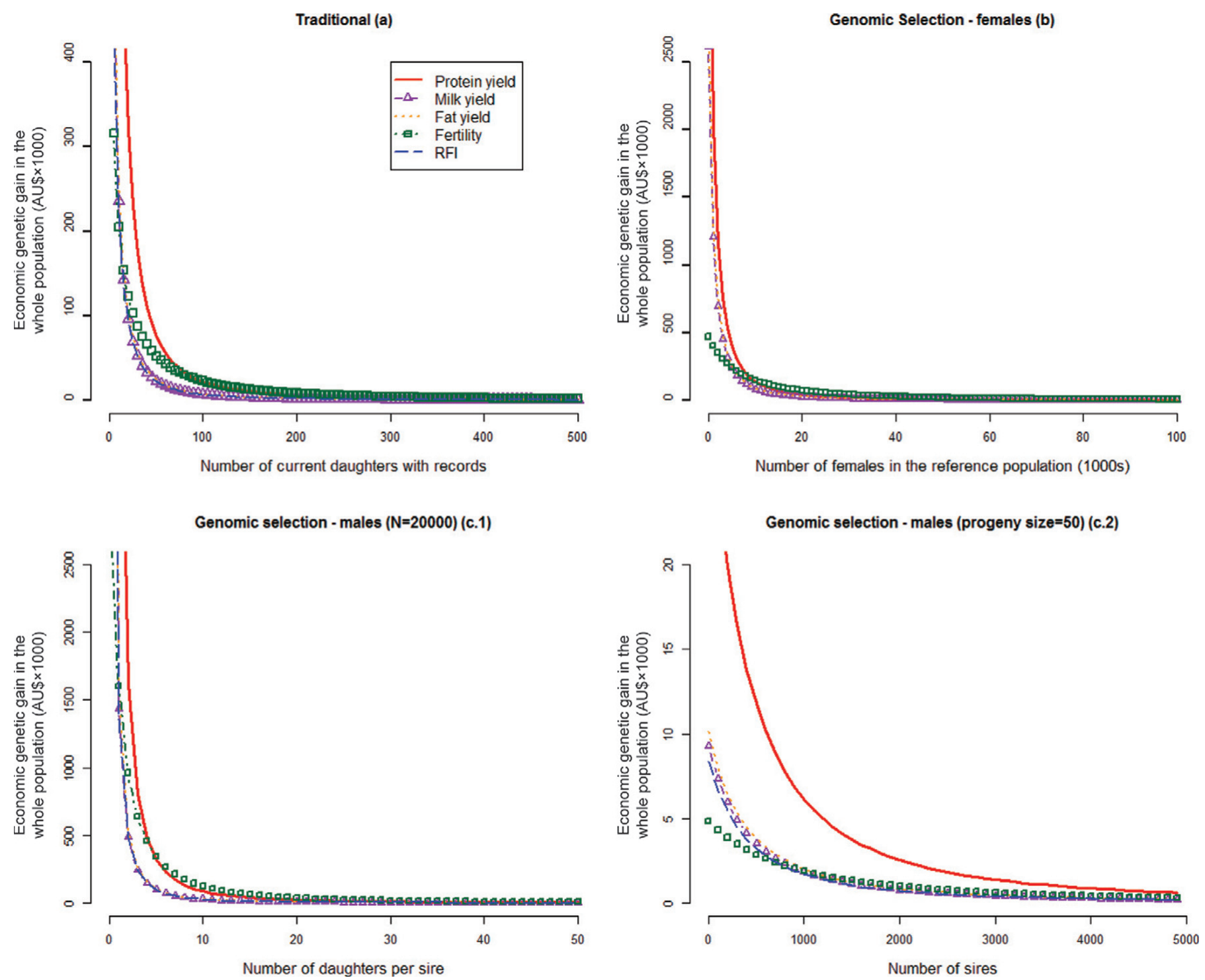

Figure 4. Economic contribution $(\$ \mathrm{AU})$ to the total Australian Holstein population of an additional phenotype given the amount of daughters per sire that have already been phenotyped for different traits [protein, milk yield, calving interval, and residual feed intake (RFI)] in a (a) traditional or (b) genomic selection breeding program. Color version available in the online PDF.

sires), increasing the number of phenotyped progeny for fertility provides larger economic benefits to the whole population than phenotypes for fat or milk yield until every sire has a progeny group size larger than $10 \mathrm{ED}$ for milk yield, fat yield, and residual feed intake, 30 ED for fertility, and $50 \mathrm{ED}$ for protein yield; however, once the progeny group size is equal to 50, increasing the number of sires in the reference population would produce larger economic benefit for productive traits.

The optimization of a data recording program must therefore evaluate both the heritability and the economic value of the traits that are measured or included in the breeding objective.

\section{Relative Cost of Female Versus Male Reference Populations}

Figure 5 shows the cost ratio of creating a genomic reference population of females versus males. In general, when the phenotyping cost is low $(<\mathrm{AU} \$ 2$ per record), sire reference populations are economically more efficient than female reference populations. Female reference populations have larger costs than reference populations of sires if the progeny size group is not too large (e.g., $20 \mathrm{ED}$ ), unless $h^{2}$ is $>0.30$ and the phenotyping cost is greater than AU $\$ 100$ per record (Figure 5a). However, if a sire reference population is constructed of larger progeny group sizes (e.g., 100 ED), female refer- 
a) GS in females vs. GS in sires (20 daugthers)

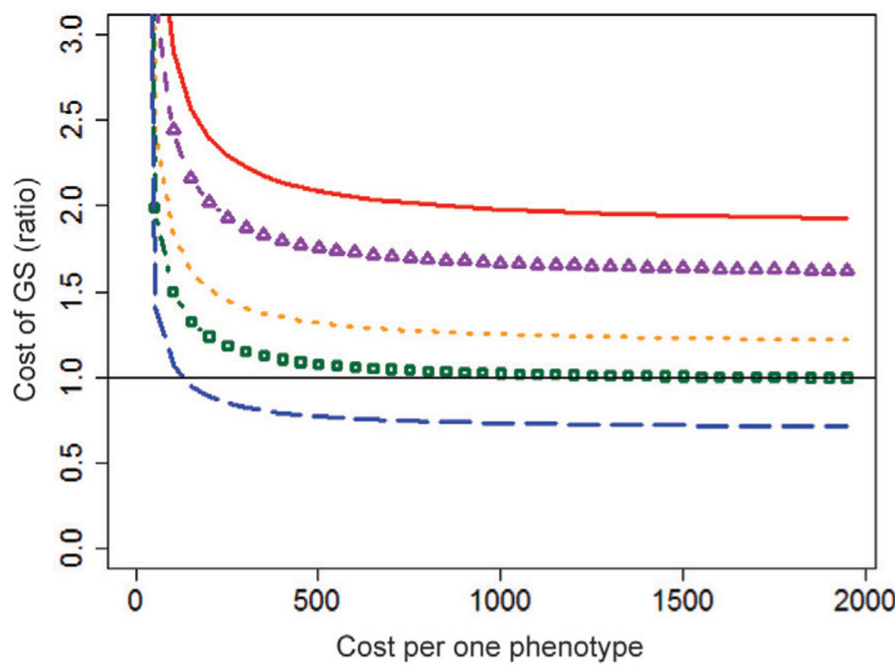

c) GS in sires (20 daugthers) vs. GS in sires (100 daugthers)

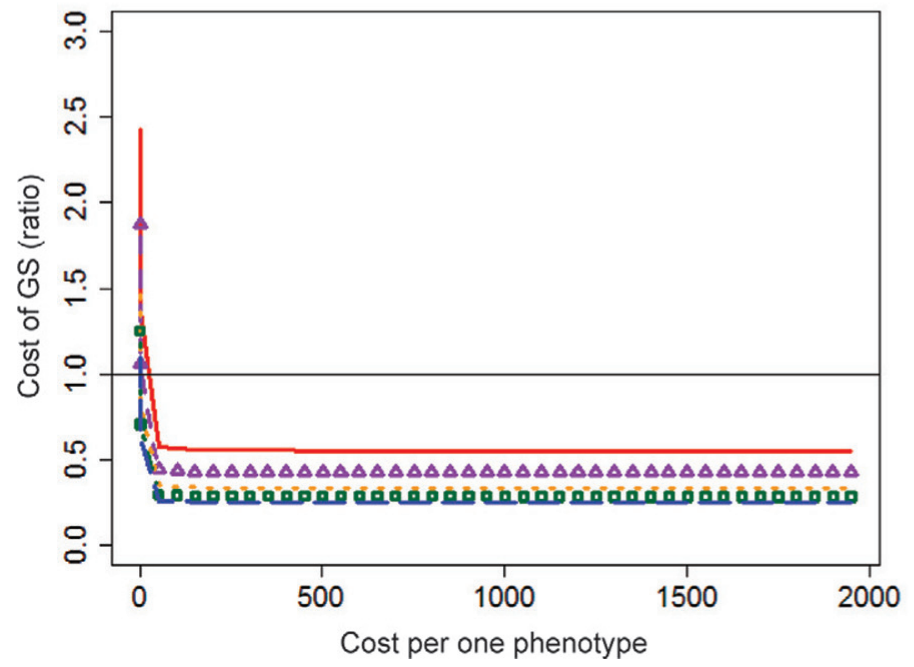

b) GS in females vs. GS in sires (100 daugthers)

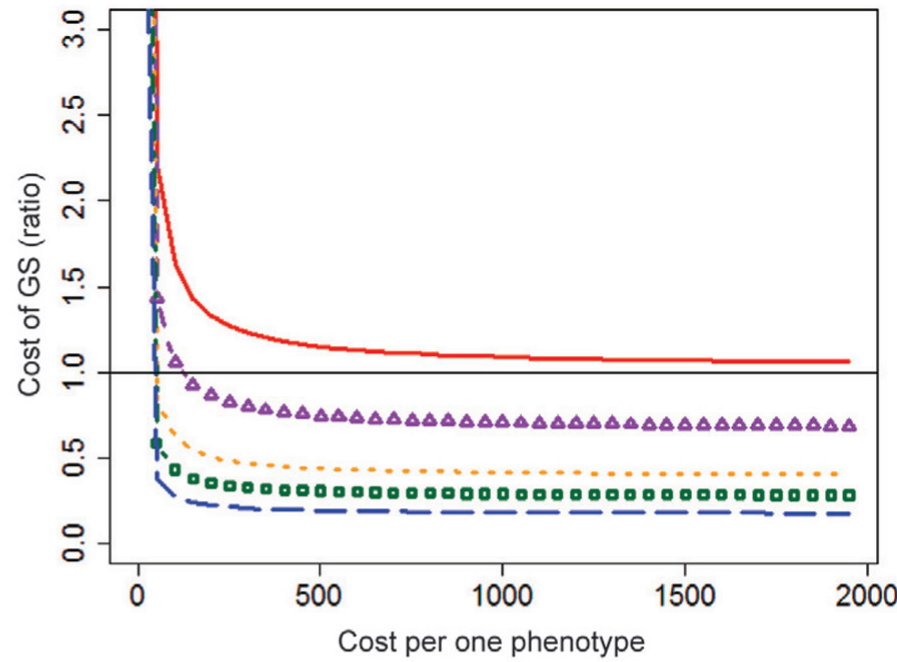

Figure 5. Relative cost of achieving a reliability of 0.60 in a genomic selection (GS) program applied in a female reference population versus a sire reference population, regarding the heritability of the trait and the cost of phenotyping. Two sire reference populations were evaluated: one with progeny size of 20 daughters and another one with progeny size of 100 daughters. Ratios $>1$ show lower cost for a sire reference population in (a) and (b), and lower cost for a sire reference population with progeny size equal to 100 (vs. 20) in (c). Color version available in the online PDF.

ence populations are more cost effective as long as the phenotypic cost is more than AU $\$ 50$ per record, and the $h^{2}$ of the trait is $>0.10$ (Figure $5 \mathrm{~b}$ ). Sire reference populations of large progeny group size (100 daughters) are the cheapest scheme when the cost of phenotyping is less than $\mathrm{AU} \$ 20$, but as the cost of phenotyping increases, smaller progeny group sizes (e.g., 20 ED) are associated with a lower cost of establishing the reference population (Figure 5c), and as the heritability of the trait and the cost of phenotyping increases, female reference populations can obtain similar predictive ability at lower cost.

\section{DISCUSSION}

Phenotypic data, in addition to pedigree records, have been the cornerstone of genetic improvement programs in agriculture. Genomic selection in dairy cattle offers new opportunities to accelerate rates of genetic gain at lower cost (Schaeffer, 2006), in addition to reducing the reliance on pedigree recording by allowing pedigree relationship matrices to be replaced with the counterpart (genomic relationships) derived from genotypes. The success of both traditional and genomic selection rely on the collection of phenotypes to provide accurate 
estimates of genetic merit, yet the value of phenotypes has largely been ignored in dairy breeding, possibly because they are collected as a by-product of MRO.

The value of a phenotype may not have important implications in breeding programs that conserve the current mode of organization or status quo; however, scenarios do exist where this may be one of the principles to be considered in a breeding program. The first is the establishment of a new breeding program where phenotype recording must be organized, and the phenotyping cost per animal is not too high for the most important traits (e.g., conformation traits, growth and yield traits). In this case, the contribution of individual phenotypes is initially high, but as the size of the progeny groups or the genomic reference population increases, the reliability and ultimately the economic contribution of each individual phenotype declines. It is especially important to record phenotypes for those traits that have the largest effect on the overall merit index. It is clear that PT programs can obtain higher reliabilities in the prediction of genetic merit than GS if only one generation is considered, but GS can be done at a lower cost than PT as long as long-term selection is considered (Schaeffer, 2006). The heritability of each trait and the number of individuals to be tested yearly must be carefully evaluated before going to either of the GS alternatives. Besides, GS has been shown to be an efficient tool in selecting animals with little or no phenotypic information such as young bulls (with few or no offspring), cows, and sires with first crop of daughters for low heritability traits (Goddard, 2009; Hayes et al., 2009). Nonetheless, broad PT may be convenient because phenotyping is available from MRO for other purposes and reliabilities offered by PT are large, mainly for low heritability traits.

The second scenario is the inclusion of new traits that are expensive or difficult to measure in the breeding objective. Smaller progeny group sizes are more efficient in this scenario, regardless of the heritability of the trait, unless the heritability of the trait and the cost of phenotyping are both high, in which case female reference populations are preferred. For instance, for a trait such as residual feed intake or methane emissions, where the cost of phenotyping is around $\mathrm{AU} \$ 1,000$ (or more) per cow and $h^{2}$ is between 0.30 and 0.50 , female reference populations are clearly more cost effective. Again, the first 50 records per sire or the first cow phenotype contribute most to the increase in reliability, and this might be considered as compensation for farmers that do phenotype recording. Nonetheless, some traits can only be phenotyped under expensive experimental conditions, and it is not worth collecting more phenotypes when no further increase in annual genetic gain is expected. For feed efficiency traits, as an example again, phenotyping is very expensive, but has large financial benefits to all farmers when used in selection decisions. Collaborations between countries and research centers are necessary in these cases to improve accuracy at a reasonable cost (Berry et al., 2014). For example, de Haas et al. (2012) showed that the accuracy of genomic prediction of DMI can be increased by combining the data from 3 research herds from 3 different countries (UK, the Netherlands, and Australia). However, accurate genomic breeding values need large reference populations and the cost versus the benefit may be questionable.

The third scenario worth mentioning is that in which the phenotype availability of traits that were traditionally routinely measured becomes scarce; for instance, due to reduced interest in milk recording, farm automation, or because affordability reduces due to other pressures. Here, the contribution of additional phenotypes is small because a large amount of historical recorded data has already been collected. However, a prolonged reduction in the availability of new phenotypes would have a negative effect on the reliability of breeding values in the future. Hence, it is necessary to ensure a continued flow of phenotypes to guarantee continued predictive ability of the reference population. This might be done by compensating farmers to ensure a minimum necessary progeny size, in ED, or the yearly update of a reference population, either male or female depending on the trait, that ensures a minimum genomic EBV reliability. Female-only reference populations are already being established, such as the Australian Genomic Information nucleus (Ginfo) with a reference population of around 30,000 females. Currently, these populations are designed to augment reference populations that consist of bulls with very reliable phenotypes based on their progeny, and are used to compensate for some aspects of phenotypic recording where data are lacking. For instance, the increased popularity of young genomic bulls (Ducrocq and Wiggans, 2014) over time may eliminate or reduce progeny testing to a minimum.

It must be noted that this comparison is valid for current methodology capturing the additive and linear contribution of the genetic predictions. Different results may be observed if nonadditive effects are included as part of predictions, because the relative contribution of the different sorts of reference population would be different under that scenario.

\section{CONCLUSIONS}

The main contribution of this study is in quantifying the value of phenotypes in GS breeding programs that have either male or female reference populations compared with a PT breeding program. The contribution of 
an additional phenotype was calculated as the expected increase in profit through genetic gain achieved in the whole population from the addition of a single record or phenotype. The main factors affecting the value of a phenotype are the economic value of the trait, the number of phenotypic records already available for the trait, and the heritability. Therefore, the cost of establishing a GS breeding program must be evaluated under particular conditions of the breeding program and its economic breeding objectives. Data recording is essential regardless of the scheme and can be used to evaluate the cost versus return of collecting phenotypes for a novel trait. When only a few phenotypes are available, the value of a new phenotype to genetic gain is much larger than when a large number of phenotypes are available. Progeny testing is the basis of both traditional and GS breeding programs; however, its size may be reduced in GS with the subsequent decrease of phenotyping costs. Breeding programs that include new traits that are expensive to measure should consider female reference populations when the $h^{2}$ of the trait under selection is $>0.30$; otherwise sire reference populations with small progeny group sizes (e.g., $20 \mathrm{ED}$ ) are better. Finally, compensation, or payment for collecting phenotypes, can be justified by the increase in profitability in the population resulting from genetics.

\section{ACKNOWLEDGMENTS}

Oscar Gonzalez-Recio and Jennie Pryce thank Department of Environment and Primary Industries (Melbourne, Australia) and Dairy Futures Cooperative Research Centre (Melbourne, Australia) for funding this project. Mike Coffey acknowledges financial support from the Scottish Government and DairyCo (Kenilworth, UK). Eileen Wall (SRUC, Edinburgh, UK) is acknowledged for contributing to discussions.

\section{REFERENCES}

Berry, D. P., M. P. Coffey, J. E. Pryce, Y. de Haas, P. Løvendahl, N. Krattenmacher, J. J. Crowley, Z. Wang, D. Spurlock, K. Weigel, K. Macdonald, and R. F. Veerkamp. 2014. International genetic evaluations for feed intake in dairy cattle through the collation of data from multiple sources. J. Dairy Sci. 97:3894-3905.

Cameron, N. D. 1997. Selection Indices and Prediction of Genetic Merit in Animal Breeding. CAB International, Wallingford, UK.

Daetwyler, H. D., B. Villanueva, and J. A. Woolliams. 2008. Accuracy of predicting the genetic risk of disease using a genome-wide approach. PLOS ONE 3:e3395.

de Haas, Y., M. P. L. Calus, R. F. Veerkamp, E. Wall, M. P. Coffey, H. D. Daetwyler, B. J. Hayes, and J. E. Pryce. 2012. Improved accuracy of genomic prediction for dry matter intake of dairy cattle from combined European and Australian data sets. J. Dairy Sci. 95:6103-6112.

Ducrocq, V., and G. Wiggans. 2014. Genetic improvement of dairy cattle. Pages 370-395 in Genetics of Cattle. 2nd ed. D. J. Garrick and A. Ruvinsky, ed. CAB International, Wallingford, UK.

Fikse, W. F., and G. Banos. 2001. Weighting factors of sire daughter information in international genetic evaluations. J. Dairy Sci. 84:1759-1767.

Goddard, M. E. 2009. Genomic selection: Prediction of accuracy and maximisation of long term response. Genetica 136:245-252.

Groen, A. F. 1990. Cattle breeding goals and production circumstances. PhD Thesis. Department of Animal Breeding, Wageningen Institute of Animal Science, Wageningen, the Netherlands.

Hayes, B. J., P. J. Bowman, A. J. Chamberlain, and M. E. Goddard. 2009. Genomic selection in dairy cattle: Progress and challenges. J. Dairy Sci. 92:433-443.

Hutchison, J. L., J. B. Cole, and D. M. Bickhart. 2014. Short communication: Use of young bulls in the United States. J. Dairy Sci. 97:3213-3220.

ICAR (International Committee on Animal Recording). 2013. Milk recording surveys for dairy cattle. Accessed May 2014. http://www. survey-icar.org/cow_survey/.

Lush, J. L. 1945. Animal Breeding Plans. 3rd ed. Iowa State University Press, Ames.

Pryce, J. E., and H. D. Daetwyler. 2012. Designing dairy cattle breeding schemes under genomic selection-A review of international research. Anim. Prod. Sci. 52:107-114.

Schaeffer, L. R. 2006. Strategy for applying genome-wide selection in dairy cattle. J. Anim. Breed. Genet. 123:218-223.

VanRaden, P. M., and G. R. Wiggans. 1991. Derivation, calculation, and use of national animal model information. J. Dairy Sci. $74: 2737-2746$ 